\title{
Towards a population of HMXB/NS microquasars as counterparts of low-latitude unidentified EGRET sources
}

\author{
M. Ribó (mribo@discovery.saclay.cea.fr) \\ Service d'Astrophysique, CEA Saclay \\ J.A. Combi \\ Universidad de Jaén \\ Instituto Argentino de Radioastronomía (IAR) \\ I.F. Mirabel \\ Service d'Astrophysique, CEA Saclay \\ Instituto de Astronomía y Física del Espacio (IAFE)
}

\begin{abstract}
The discovery of the microquasar LS 5039 well within the $95 \%$ conficence contour of the Unidentified EGRET Source (UES) 3EG J1824-1514 was a major step towards the possible association between microquasars (MQs) and UESs. The recent discovery of precessing relativistic radio jets in LS I +61 303, a source associated for long time with 2CG 135+01 and with the UES 3EG J0241+6103, has given further support to this idea. Finally, the very recently proposed association between the microquasar candidate AX J1639.0-4642 and the UES 3EG J1639-4702 points towards a population of High Mass X-ray Binary (HMXB)/Neutron Star (NS) microquasars as counterparts of low-latitude unidentified EGRET sources.
\end{abstract}

Keywords: $\gamma$-ray sources, X-ray binaries, microquasars

\section{Introduction}

The third EGRET catalog (Hartman et al. 1999) contains 271 point sources detected at energies above $100 \mathrm{MeV}$. The majority of these sources, $\sim 168$ or $\sim 62 \%$, still remain unidentified. Among them, there are 72 sources located at low galactic latitudes, having $|b|<10^{\circ}$, which represents around $45 \%$ of the UES population. Therefore, several of these objects are presumably of galactic nature. Similar properties between some of these UESs, indicate that there are at least three different groups of galactic populations (Romero et al. 2004, Grenier 2004). The group of young stellar objects and star-forming regions (Romero 2001), those sources forming a halo around the galactic center and a group of sources correlated with the Gould Belt (Grenier 2000).

Based both on multiwavelength observations and theory, microquasars (see Mirabel \& Rodríguez 1999 for a review) with massive companions have been proposed as possible counterparts of the first group of galactic UESs by several authors (Paredes et al. 2000, Kaufman Bernadó et al. 2002, Romero et al. 2004, Bosch-Ramon et al. 2004). In Sects. 2

(C) 2013 Kluwer Academic Publishers. Printed in the Netherlands. 

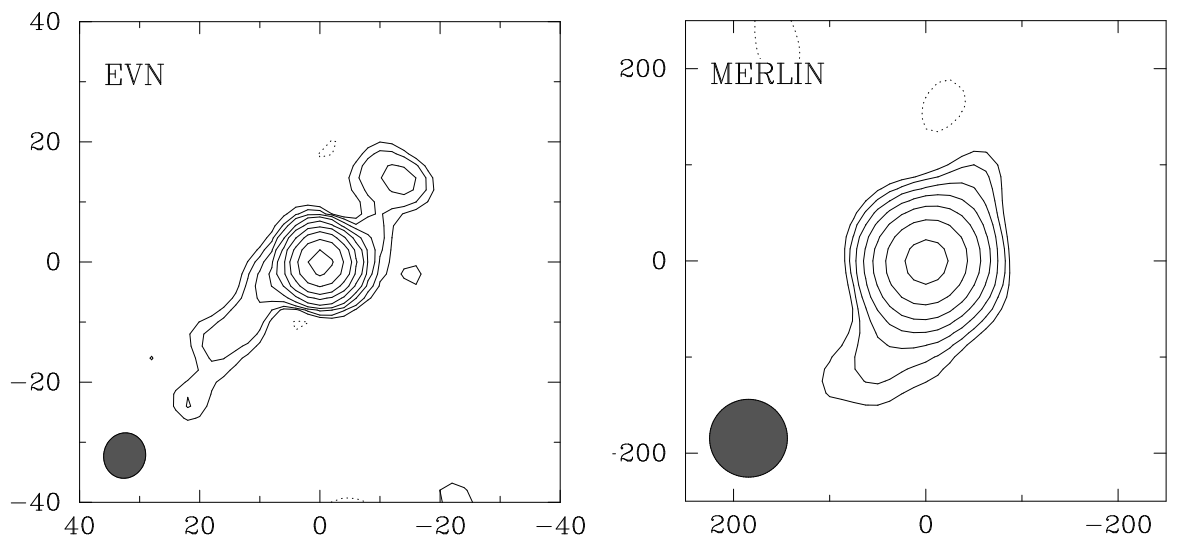

Figure 1. Left: EVN self-calibrated image of LS 5039 at $5 \mathrm{GHz}$ obtained on 2000 March 1. Axes units are in mas. The synthesized beam is plotted in the lower left corner. Right: Same as left but obtained with MERLIN. From Paredes et al. (2002).

and 3 of this paper we will briefly review the properties of the two wellknown microquasars LS 5039 and LS I +61 303, typically associated with the first group of UESs, while in Sect. 4 we will present the possible association between the microquasar candidate AX J1639.0-4642 and the UES 3EG J1639-4702. Finally, in Sect. 5 we will compare the available data of these 3 sources from radio to gamma-rays, and we will discuss on similarities pointing towards a population of HMXB with NS microquasars as counterparts of low-latitude UESs.

\section{LS 5039}

The high mass X-ray binary system LS 5039 (Paredes et al. 2000) is one of the $\sim 15$ confirmed galactic microquasars (Ribó 2004). LS 5039

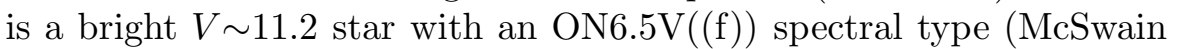
et al. 2004). The binary system has a short orbital period of $P=$ $4.4267 \pm 0.0005 \mathrm{~d}$, a high eccentricity of $e=0.48 \pm 0.06$, and a low mass function $f(m)=0.0017 \pm 0.0005 M_{\odot}$, suggesting the presence of a NS as the compact object in the system (McSwain et al. 2004).

Observations conducted with the EVN and MERLIN (see Fig. 1) confirmed the persistent nature of this MQ, and revealed the presence of an asymmetric two-sided jet reaching up to $1000 \mathrm{AU}$ on the longest jet arm (Paredes et al. 2002). These observations also suggest a bending of the jets with increasing distance from the core and/or precession.

The possibility that LS 5039 is a $\gamma$-ray emitter was suggested by Paredes et al. (2000), who proposed the association of the system with the UES 3EG J1824-1514 (Hartman et al. 1999). We show in 


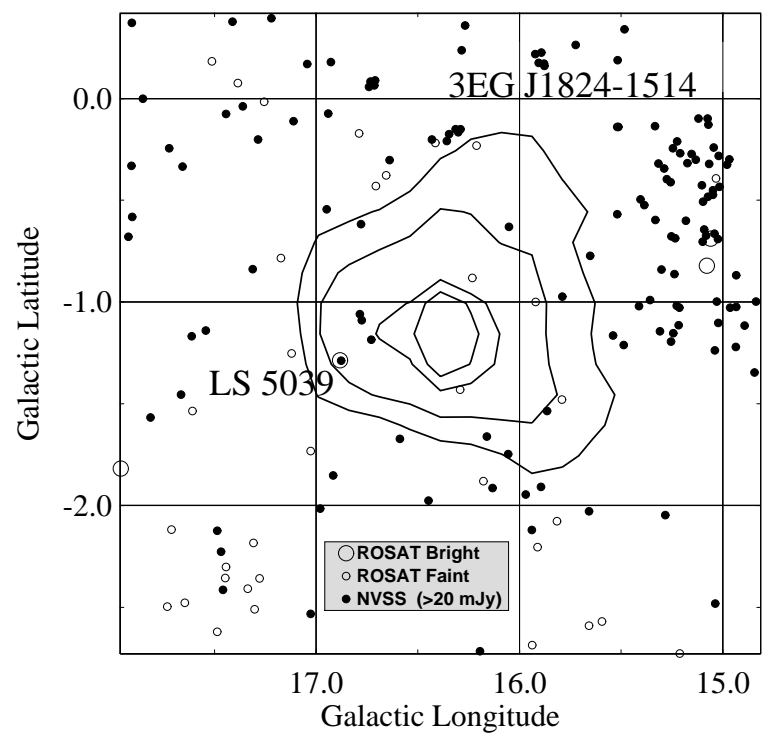

Figure 2. Location map of 3EG J1824-1514. The contours represent, from inside to outside, the $50 \%, 68 \%, 95 \%$, and $99 \%$ statistical probability that a $\gamma$-ray source lies within the given contour. The only source with X-ray and radio emission (filled circle inside an open big circle, $l=16.88^{\circ}$ and $\left.b=-1.29^{\circ}\right)$, and well inside the $95 \%$ contour, is LS 5039 (Ribó 2002).

Fig. 2 the location map of the $\gamma$-ray source together with the NVSS and bright/faint ROSAT sources. The only simultaneous X-ray/radio source within the statistical contours of 3EG J1824-1514 is the microquasar LS 5039. We note that this binary system is present in the BATSE Earth occultation catalog of low-energy gamma-ray sources (Harmon et al. 2004), with a positive detection of a few mCrab up to $\sim 100 \mathrm{keV}$. The source is not present in cumulative observations conducted with the INTEGRAL satellite (Bird et al. 2004), although it is expected to be detected when adding a few more months of data. We also point out that there is an unidentified COMPTEL source with a position compatible with LS 5039 (Collmar 2004).

Astrometric studies carried out by Ribó et al. (2002), show that it is a runaway system with a systemic velocity of $\sim 150 \mathrm{~km} \mathrm{~s}^{-1}$ that moves away from the Galactic plane with a velocity of $\sim 100 \mathrm{~km} \mathrm{~s}^{-1}$. This result, combined with the possible lifetime of the donor star, indicates that it could reach a not-so-low galactic latitude of $b=-12^{\circ}$ still behaving as a microquasar.

Bosch-Ramon \& Paredes (2004a) have recently developed a detailed numerical model to test whether this system can actually produce the emission detected by EGRET through inverse Compton (IC) scattering. Their numerical approach considers a population of relativistic 


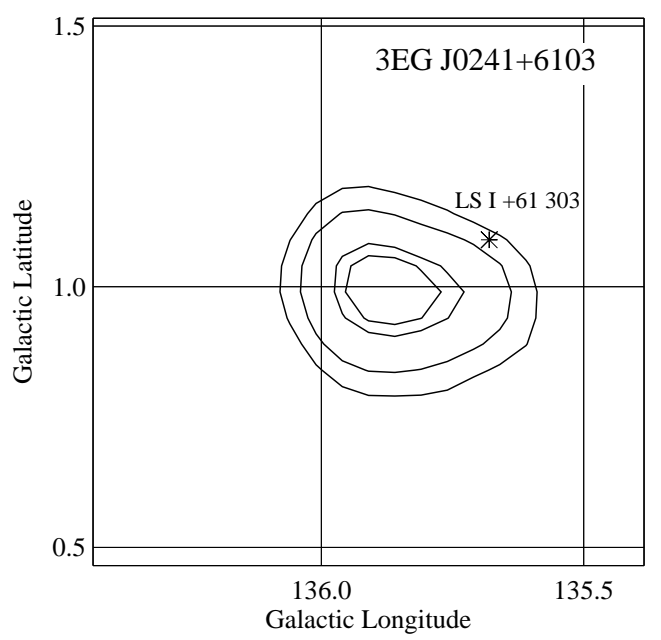

Figure 3. Location map of 3EG J0241+6103. The position of LS I +61 303 is marked with an asterisk. Adapted from Hartman et al. (1999).

electrons entrained in a cylindrical inhomogeneous jet, which interact with both the radiation and the magnetic fields. The computed spectrum is able to reproduce the observed spectral characteristics at very high $(\mathrm{GeV})$ energies.

\section{LS I +61 303}

The Be/X-ray binary system LS I +61 303 is a well-studied object since it presents radio and X-ray variability linked to its $\sim 26.5 \mathrm{~d}$ orbital period (Gregory 2002; Paredes et al. 1997). The donor star in this system is a rapidly rotating B0V star with variable mass loss (Hutchings \& Crampton 1981). Some properties of this system can be explained assuming that the unseen companion is a non-accreting young pulsar with a relativistic wind strongly interacting with the wind of the Be star (Maraschi \& Treves 1981). On the contrary, other properties of LS I +61 303 fit better a model where the companion is accreting even with two episodes of super-critical accretion along the orbit (Martí \& Paredes 1995).

This X-ray binary system has been associated for long time with the gamma-ray source 2CG 135+01/3EG J0241+6103 (see Fig. 3), which displays variability on timescales of days (Tavani et al. 1996, 1998; Wallace et al. 2000). During the last years, Massi et al. (2001, 2004) have revealed its MQ nature through the discovery of a radio jet (see Fig. 4) extending $200 \mathrm{AU}$ at both sides of a central core, 

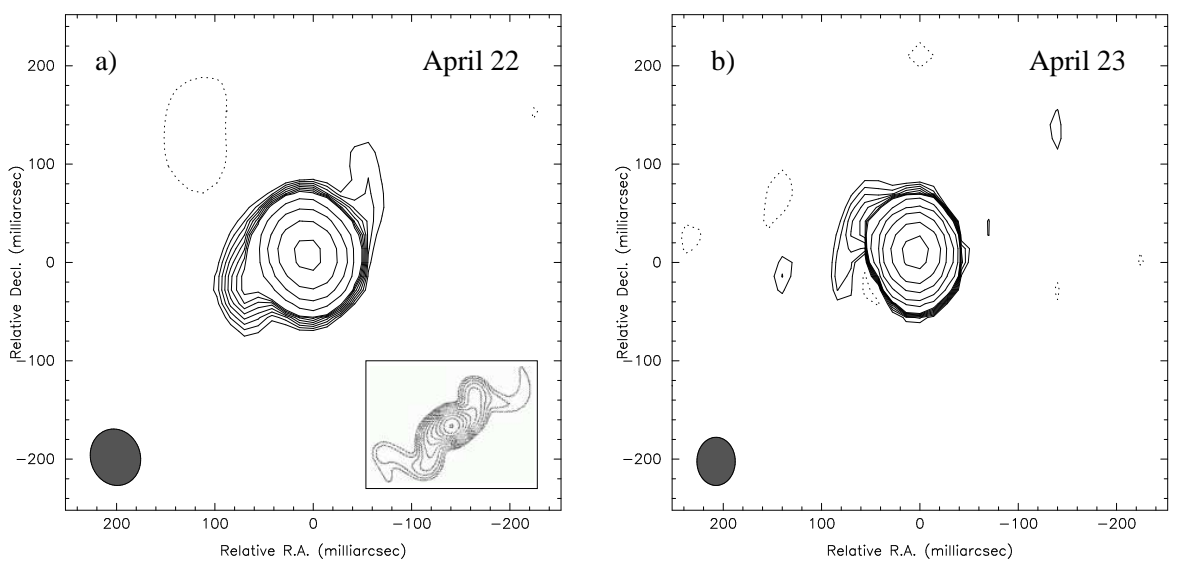

Figure 4. a) MERLIN self-calibrated image of LS I +61303 at $5 \mathrm{GHz}$ and using natural weights, obtained on 2001 April 22. The synthesized beam is plotted in the lower left corner. The S-shaped morphology strongly recalls the precessing jet of SS 433, whose simulated radio emission (Fig. 6b in Hjellming \& Johnston 1988, rotated here for comparison purposes) is given in the small box. b) Same as before but for the April 23 run and using uniform weights for better displaying. From Massi et al. (2004).

that appears to experience a fast precession, which could explain the short-term gamma-ray variability of 3EG J0241+6103 (as proposed by Kaufman Bernadó et al. 2002) and the puzzling VLBI structures found in previous observations. This result points to the occurrence of accretion/ejection processes in this system, ruling out, in principle, the non-accreting young pulsar scenario.

Massi (2004) has recently studied the data acquired within the pointed EGRET observations of 3EG J0241+6103 and claimed the detection of a periodicity of $P=27.4 \pm 7.2 \mathrm{~d}$, consistent with the orbital period of the binary system. If this is confirmed, the identification of LS I +61 303 as the counterpart of 3EG J0241+6103 would be unambiguous. In any case, an important point is that all the available data are compatible with an increase of $\gamma$-ray emission around periastron, that can be tested with Cherenkov telescopes and future satellites.

This binary system is also present in the BATSE Earth occultation catalog of low-energy gamma-ray sources (Harmon et al. 2004), with a positive detection of a few mCrab up to $\sim 100 \mathrm{keV}$, although the detection is not as significant as in the case of LS 5039. LS I +61 303 is not present in cumulative observations conducted with the INTEGRAL satellite (Bird et al. 2004), although it is expected to be detected when adding a few more months of data, like in the case of LS 5039. We note that there is a COMPTEL source containing LS I +61 303 and the quasar QSO 0241+622 (van Dijk et al. 1996). 


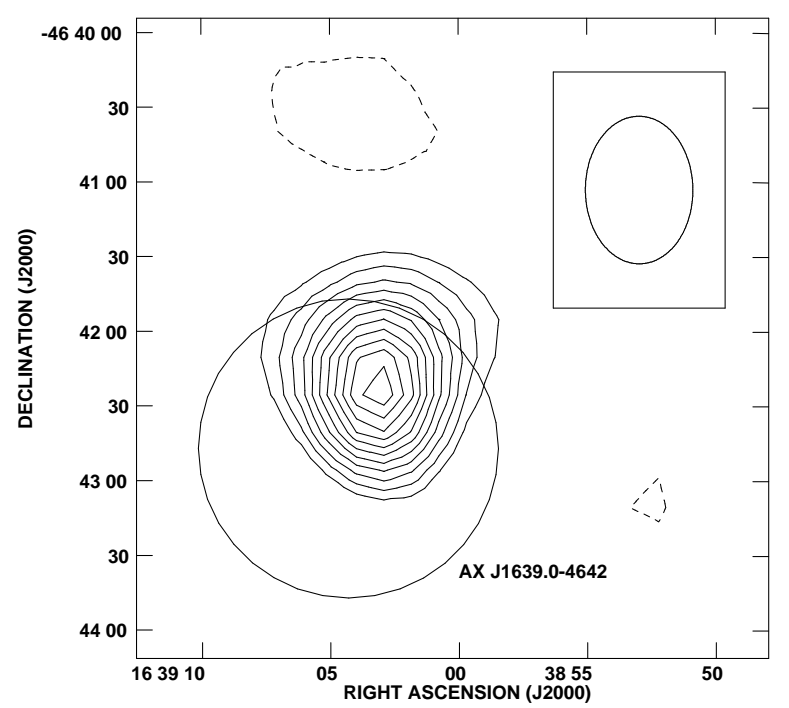

Figure 5. Contour image of the MGPS data obtained with MOST at $843 \mathrm{MHz}$ on 1992 April 16. The radio source MOST J1639.0-4642 is well within the $90 \%$ uncertainty error circle of the X-ray source AX J1639.0-4642. The ellipse in the top right corner is the convolving beam. From Combi et al. (2004).

A numerical model to explain the EGRET emission of LS I +61 303 has also been developed by Bosch-Ramon \& Paredes (2004b).

\section{AX J1639.0-4642}

Aimed at discovering new MQs, Combi et al. (2004) have recently carried out a multiwavelength study of the unidentified X-ray source AX J1639.0-4642. This object was discovered by the Advanced Satellite for Cosmology and Astrophysics (ASCA) observatory at the 0.7$10 \mathrm{keV}$ energy range, and presented as a possible HMXB (Sugizaki et al. 2001). Its measured flux was $F_{\mathrm{X}(0.7-10 \mathrm{keV})}=19.2 \times 10^{-12} \mathrm{erg} \mathrm{cm}^{-2} \mathrm{~s}^{-1}$, and it showed variable $\mathrm{X}$-ray emission, with a confidence $\geq 99 \%$. Its spectrum was fitted with a power law with a very hard photon index $\Gamma=-0.01_{-0.60}^{+0.66}$ and a poorly constrained hydrogen column density of $N_{\mathrm{H}}=12.82_{-6.88}^{+8.58} \times 10^{22} \mathrm{~cm}^{-2}$. Combi et al. (2004) re-analyzed these data and found evidences for variability on timescales of hours.

In searching for radio sources in the field of AX J1639.0-4642, Combi et al. (2004) found that the Molonglo Galactic Plane Survey (MGPS) at $843 \mathrm{MHz}$ (Green et al. 1999) revealed a point-like radio source (see Fig. 5), dubbed MOST J1639.0-4642, well within the error box of the X-ray source, with a flux density of $136 \pm 18 \mathrm{mJy}$. At near 


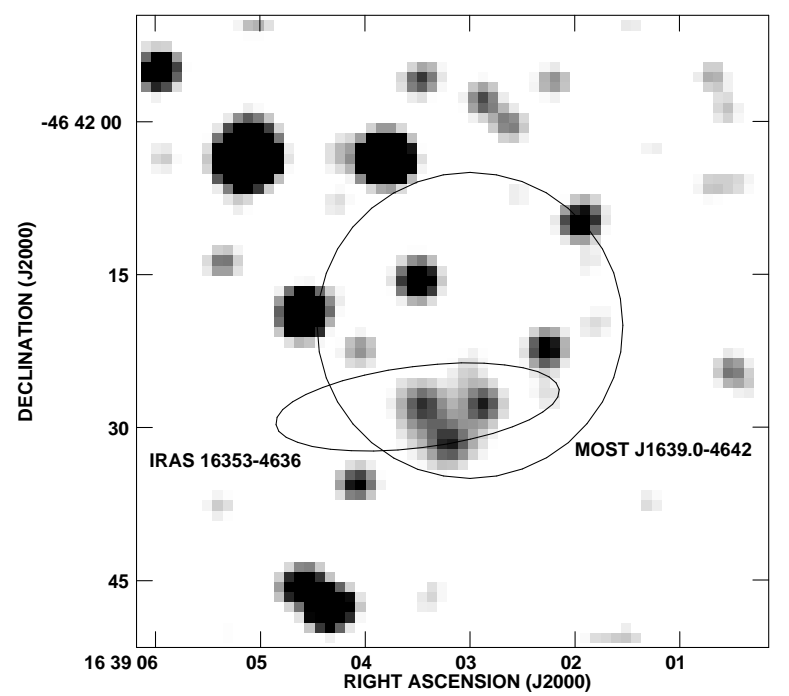

Figure 6. 2MASS $K_{\mathrm{s}}$-band image of the environment of the radio source MOST J1639.0-4642, whose $3 \sigma$ position error circle is shown, together with the $2 \sigma$ position error ellipse of the far infrared source IRAS 16353-4636. Several NIR sources from the 2MASS catalog are possible counterparts of both sources. From Combi et al. (2004).

infrared (NIR) wavelengths Combi et al. (2004) inspected the 2 Micron All Sky Survey (2MASS, Cutri et al. 2003), and found 10 sources in the $3 \sigma$ error circle in position of MOST J1639.0-4642, some of them visible in the $K_{s}$-band image shown in Fig. 6. At the far infrared part of the spectrum, from 12 to 100 microns, they found that the source IRAS 16353-4636 lies inside the error box of the X-ray source. This source overlaps the southern part of the $3 \sigma$ position error circle of MOST J1639.0-4642, and its location uncertainty ellipse contains several 2MASS sources, as can be seen in Fig. 6. The X-ray source AX J1639.0-4642 has been recently re-discovered at higher energies with the IBIS telescope onboard the INTEGRAL satellite, dubbed IGR J16393-4643 (Malizia et al. 2004; Bird et al. 2004). This source shows an average flux of $F_{\mathrm{X}(20-100 \mathrm{keV})} \simeq 5 \times 10^{-11} \mathrm{erg} \mathrm{cm}^{-2} \mathrm{~s}^{-1}$, and presents a factor of 2-3 flux variability on timescales of months.

Although there is no spectroscopic/photometric information of a NIR/optical counterpart to derive a distance to the source, assuming that it is located in the Scutum-Crux or in the Norma spiral arms, a range of distances between 3 and $13 \mathrm{kpc}$ is obtained.

Combi et al. (2004) pointed out that AX J1639.0-4642 lies inside the 95\% location contour of the UES 3EG J1639-4702 (Hartman et al. $1999)$ as can be seen in Fig. 7. Its $\gamma$-ray flux is $(53.2 \pm 8.7) \times 10^{-8}$ photon $\mathrm{cm}^{-2} \mathrm{~s}^{-1}$, presents a steep $\gamma$-ray spectral index of $\Gamma=2.5 \pm$ 


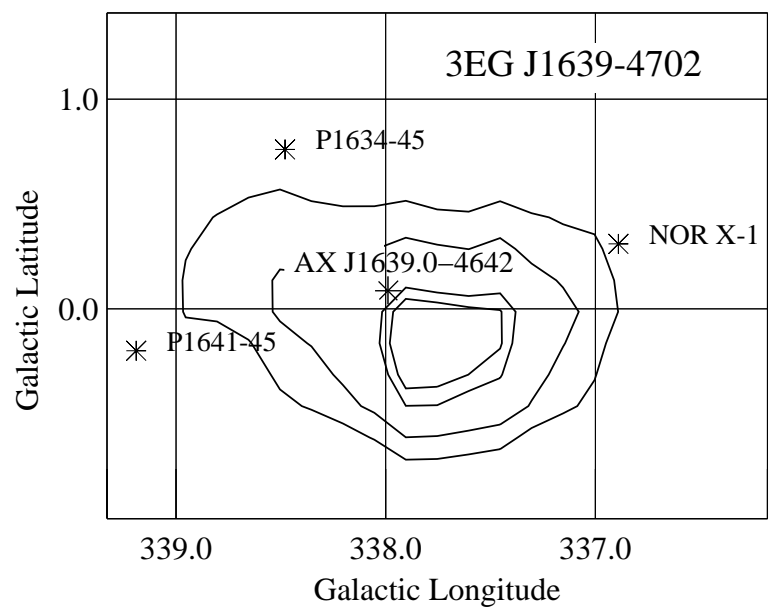

Figure 7. Location map of 3EG J1639-4702. The position of the X-ray source AX J1639.0-4642, well within the $95 \%$ probability contour, is marked with an asterisk. Other sources appearing outside the $99 \%$ probability contour are also indicated. From Combi et al. (2004).

0.18 and has a variability index of $I=1.95$. Although Torres et al. (2001a) found three radio pulsars inside the 95\% confidence contour of the $\gamma$-ray source, its possible variability and steep photon index do not seem to agree, in principle, with a pulsar origin. Similarly, these properties would rule out an association with the three SNRs found within the 95\% confidence contour (Torres et al. 2003). Moreover, no identified blazar has been found within the $\gamma$-ray contours. Therefore, Combi et al. (2004) suggested that the microquasar candidate AX J1639.0-4642/MOST J1639.0-4642 (=IGR J16393-4643) is the counterpart of 3EG J1639-4702. Observations with ATCA are in progress to unveil the nature of this source.

\section{HMXB/NS microquasars as counterparts of low-latitude unidentified EGRET sources}

As discussed above, the possibility of MQs being $\gamma$-ray emitters was suggested by Paredes et al. (2000), who proposed the association between the HMXB LS 5039 and the UES 3EG J1824-1514. In their scenario (Paredes et al. 2000, 2002) the $\gamma$-rays are produced by IC upscattering of stellar ultraviolet (UV) photons by the non-thermal relativistic electron population that later on will produce the detected radio emission. Recently, more detailed models considering precession (Kaufman Bernadó et al. 2002), hadronic jets in windy microquasars (Romero et al. 2003) and all possible photon fields (Bosch-Ramon 
Table I. Properties of the three $\gamma$-ray sources discussed in the text. The luminosity interval for AX J1639.0-4642 reflects the range of assumed possible distances.

\begin{tabular}{lcccc}
\hline$\gamma$-ray source & $\Gamma_{\gamma}{ }^{a}$ & $I^{b}$ & $\begin{array}{c}L_{\gamma(>100 \mathrm{MeV})}{ }^{c} \\
\left(\mathrm{erg} \mathrm{s}^{-1}\right)\end{array}$ & $\begin{array}{c}d \\
(\mathrm{kpc})\end{array}$ \\
\hline 3EG J1824-1514 & $2.19 \pm 0.18$ & 3.00 & $3.6 \times 10^{35}$ & $2.9^{d}$ \\
3EG J0241+6103 & $2.21 \pm 0.07$ & 1.31 & $3.1 \times 10^{35}$ & $2.0^{e}$ \\
3EG J1639-4702 & $2.50 \pm 0.18$ & 1.95 & $(3-50) \times 10^{35}$ & $3-13 ?$ \\
\hline${ }^{a}$ Hartman et al. (1999); ${ }^{b}$ Torres et al. (2001b); ${ }^{c}$ computed using the \\
photon fluxes and indices from Hartman et al. (1999); ${ }^{d}$ Ribó et al. (2002); \\
Frail \& Hjellming (1991).
\end{tabular}

Table II. Proposed X-ray/optical/radio counterparts of the three $\gamma$-ray sources of Table I. In the cases of the microquasars LS 5039 and LS I +61 303 the luminosity intervals correspond to intrinsic variability of the sources at the corresponding distances, while in the case of AX J1639.0-4642 they reflect the range of assumed possible distances.

\begin{tabular}{|c|c|c|c|c|c|}
\hline X-ray source & $\begin{array}{l}L_{\mathrm{X}}(0.7-10 \mathrm{keV}) \\
\quad\left(\operatorname{erg~s}^{-1}\right)\end{array}$ & $\begin{array}{c}\left.L_{\text {radio }(0.1-100} \mathrm{GHz}\right) \\
\left(\operatorname{erg~s}^{-1}\right)\end{array}$ & $\begin{array}{l}\text { Spectral } \\
\text { type }\end{array}$ & $\begin{array}{l}P_{\text {orb }} \\
\text { (days) }\end{array}$ & $\begin{array}{c}d \\
(\mathrm{kpc})\end{array}$ \\
\hline LS 5039 & $(0.5-5) \times 10^{34 a}$ & $\sim 1.0 \times 10^{31 b}$ & ON6.5V((f)) ${ }^{c}$ & $4.4^{c}$ & $2.9^{d}$ \\
\hline LS I +61303 & $(1-6) \times 10^{34 e}$ & $(1-17) \times 10^{31 f}$ & $\mathrm{BOVe} \mathrm{e}^{g}$ & $26.5^{h}$ & $2.0^{i}$ \\
\hline AX J1639.0-4642 & $(2-40) \times 10^{34}$ & $(0.8-16) \times 10^{31 j}$ & $?$ & $?$ & $3-13 ?$ \\
\hline
\end{tabular}

et al. 2004) have been proposed to explain the high-energy gamma-ray emission from HMXB microquasars.

On the other hand, as already stated, the X-ray binary system LS I +61 303 has been associated with the UES 3EG J0241+6103, and Massi et al. (2001, 2004) have revealed its MQ nature. If the MQ nature of AX J1639.0-4642 is confirmed, it could be the third MQ source related to a UES.

We quote the basic properties of these three $\gamma$-ray sources in Table I, and the properties of the proposed X-ray counterparts in Table II. There are 3 observational facts that should be noted. The first one is that LS 5039 and LS I +61 303, and probably AX J1639.0-4642, have massive optical companions, which provide an intense stellar UV photon field. On the other hand, the compact object appears to be compatible with a neutron star in the cases of LS 5039 (McSwain et al. 2004) and LS I +61 303 (Hutchings \& Crampton 1981; Casares et al, 2004; but see Massi 2004). Finally, it is interesting to point out that the luminosities obtained in each spectral domain are very similar in all three 
sources, specially for the shorter distances to AX J1639.0-4642, giving support to the idea that all of them have similar emission processes.

Moreover, it should be noted that LS 5039 and LS I +61 303 are the only microquasars having both a high-mass donor and (possibly) a NS as the compact object, and that there are no other microquasars (containing low-mass donors and/or black holes) located within the probability contours of unidentified EGRET sources. Therefore, a strong statement can be made: HMXB/NS microquasars appear as good counterparts of low-latitude unidentified EGRET sources.

This statement is followed by some natural questions. 1) May LS 5039 and LS I +61 303 still contain black holes (BHs)? Although formally possible, this is unlikely, because regarding the properties of their X-ray emission they should be in the so-called low/hard state (Fender \& Maccarone 2004), but they do not follow the empirical correlation between X-ray and radio flux found by Gallo et al. (2003), in the sense that they are clearly too radio loud. 2) Why no HMXB microquasars with BHs (e.g., Cygnus X-1) are present in the third EGRET catalog? A possibility is that black hole state changes may play a role, preventing the detection of gamma-ray emission when the jet is not present during the high/soft state (but this state is very rare in the case of Cygnus X-1 and in fact, as discussed by Romero et al. (2002), a low high-energy cutoff of a few hundreds in the Lorentz factor of the electrons is compatible with the data). 3) Why no Low Mass X-ray Binary (LMXB) microquasars are present in the third EGRET catalog? One possibility is that the optical companions do not provide the necessary intense UV radiation fields needed for an effective IC process to produce high-energy gammarays. This possibility is strongly model dependent, and not valid if Self Synchrotron Compton losses are dominant. The other possibility is that LMXBs are in general terms transient objects, that maybe were not active during the EGRET viewing periods, while HMXBs tend to be persistent systems. 4) Of course, BH and LMXB microquasars could still emit high-energy gamma-rays and not be present in the third EGRET catalog because of the relatively poor sensitivity threshold of the instrument.

In conclusion, persistent HMXBs containing NSs not experiencing state changes are good candidates for the counterparts of the still unidentified high-energy gamma-ray sources at low-galactic latitudes (approximately up to $|b|<10^{\circ}$, as discussed for LS 5039), and we consider that these objects may define a population among UESs. Observations with the future missions AGILE and GLAST will confirm or reject both the proposed associations between these microquasars and the corresponding high-energy gamma-ray sources, and the hypothesis discussed above. 


\section{Acknowledgements}

We thank Sylvain Chaty, Paula Benaglia, Gustavo E. Romero, Josep M. Paredes, Josep Martí, Valentí Bosch-Ramon and Rob Fender for useful discussions, and an anonymous referee for useful comments that helped to improve the paper. M.R. acknowledges support by a Marie Curie Fellowship of the European Community programme Improving Human Potential under contract number HPMF-CT-2002-02053. M.R. also acknowledges partial support by DGI of the Ministerio de Ciencia y Tecnología (Spain) under grant AYA2001-3092, as well as partial support by the European Regional Development Fund (ERDF/FEDER). J.A.C. is a researcher of the programme Ramón y Cajal funded by

the Spanish Ministery of Science and Technology and the University of Jaén. J.A.C. was supported by CONICET (under grant PEI 6384/03).

\section{References}

Bird, A. J., Barlow, E. J.; Bassani, L., et al. 2004, ApJ, 607, L33

Bosch-Ramon, V., \& Paredes, J. M. 2004a, A\&A, 417, 1075

Bosch-Ramon, V., \& Paredes, J. M. 2004b, A\&A, in press [arXiv:astro-ph/0407016]

Bosch-Ramon, V., Romero, G. E., \& Paredes, J. M. 2004, A\&A, submitted [arXiv: astro-ph/0405017]

Casares, J., Ribas, I., Paredes, J. M., \& Martí, J. 2004, MNRAS, submitted

Collmar, W. 2004, these proceedings

Combi, J. A., Ribó, M., Mirabel, I. F., \& Sugizaki, M. 2004, A\&A, 422, 1031

Cutri, R. M., Skrutskie, M. F., van Dyk, S., et al. 2003, VizieR Online Data Catalog, II/246 (http://cdsweb.u-strasbg.fr/viz-bin/Cat?II/246)

Fender, R. P., \& Maccarone, T. 2004, in Cosmic Gamma-Ray Sources, eds. K. S. Cheng and G. E. Romero, Kluwer Academic Publishers, Dordrecht, in press, [astro-ph/0310538]

Frail, D. A., \& Hjellming, R. M. 1991, AJ, 101, 2126

Gallo, E., Fender, R. P., \& Pooley, G. G. 2003, MNRAS, 344, 60

Green, A. J., Cram, L. E., Large, M. I., \& Ye, T. 1999, ApJS, 122, 207

Gregory, P. C. 2002, ApJ, 575, 427

Grenier, I. A. 2000, A\&A, 364, L93

Grenier, I. A. 2004, in Cosmic Gamma-Ray Sources, eds. K. S. Cheng and G. E. Romero, Kluwer Academic Publishers, Dordrecht, in press

Harmon, B. A., Wilson, C. A., Fishman, G. J., et al. 2004, ApJS, in press, [astro$\mathrm{ph} / 0404453]$

Hartman, R. C., Bertsch, D. L., Bloom, S. D., et al. 1999, ApJS, 123, 79

Hjellming, R. M., \& Johnston, K. J. 1988, ApJ, 328, 600

Hutchings, J. B., \& Crampton, D. 1981, PASP, 93, 486

Kaufman Bernadó, M. M., Romero, G. E., \& Mirabel, I. F. 2002, A\&A, 385, L10

Malizia, A., Bassani, L., Di Cocco, G., et al. 2004, ATel, 227

Maraschi, L., \& Treves, A. 1981, MNRAS, 194, 1P

Martí, J., \& Paredes, J. M. 1995, A\&A, 298, 151 
Martí, J., Paredes, J. M., \& Ribó, M. 1998, A\&A, 338, L71

Massi, M. 2004, A\&A, 422, 267

Massi, M., Ribó, M., Paredes, J. M., Peracaula, M., \& Estalella, R. 2001, A\&A, 376, 217

Massi, M., Ribó, M., Paredes, J. M., et al. 2004, A\&A, 414, L1

McSwain, M. V., Gies, D. R., Huang, W., et al. 2004, ApJ, 600, 927

Mirabel, I. F., \& Rodríguez, L. F. 1999, ARA\&A, 37, 409

Paredes, J. M., Martí, J., Peracaula, M., \& Ribó, M. 1997, A\&A, 320, L25

Paredes, J. M., Martí, J., Ribó, M., \& Massi, M. 2000, Science, 288, 2340

Paredes, J. M., Ribó, M., Ros, E., Martí, J., \& Massi, M. 2002, A\&A, 393, L99

Reig, P., Ribó, M., Paredes, J. M., \& Martí, J. 2003, A\&A, 405, 285

Ribó, M. 2002, PhD Thesis, Universitat de Barcelona

Ribó, M. 2004, in ASP Conference Series: "Future Directions in High Resolution Astronomy: A Celebration of the 10th Anniversary of the VLBA", J. D. Romney \& M. J. Reid (eds.), [astro-ph/0402134]

Ribó, M., Reig, P., Martí, J., \& Paredes, J. M. 1999, A\&A, 347, 518

Ribó, M., Paredes, J. M., Romero, G. E., et al. 2002, A\&A, 384, 954

Romero, G. E. 2001, in Proc. of The Nature of Unidentified Galactic High-energy Gamma-ray Sources, ed. A. Carramiñana, O. Reimer, and D. J. Thompson, ASSL Series of Kluwer Academic Publishers 51, p. 65

Romero, G. E., Kaufman Bernadó, M. M., \& Mirabel, I. F. 2002, A\&A, 393, L61

Romero, G. E., Torres, D. F, Kaufman Bernadó, M. M., \& Mirabel, I. F. 2003, A\&A, 410, L1

Romero, G. E., Grenier, I. A., Kaufman Bernadó, M., Mirabel, I. F., \& Torres, D. F. 2004, in proceedings of the 'Fifth INTEGRAL workshop The Integral Universe', ESA SP, in press [astro-ph/0402285]

Strickman, M. S., Tavani, M., Coe, M. J., et al. 1998, ApJ, 497, 419

Sugizaki, M., Mitsuda, K., Kaneda, H., et al. 2001, ApJS, 134, 77

Tavani, M., Hermsen, W., van Dijk, R., et al. 1996, A\&AS, 120, 243

Tavani, M., Kniffen, D., Mattox, J. R., Paredes, J. M., \& Foster, R. S. 1998, ApJ, 497, L81

Torres, D. F., Butt, Y. M., \& Camilo, F. 2001a, ApJ, 560, L155

Torres, D. F., Romero, G. E., Combi, J. A., et al. 2001b, A\&A, 370, 468

Torres, D. F., Romero, G. E., Dame, T. M., Combi, J. A., \& Butt, Y. M. 2003, Phys. Rep., 382, 303

van Dijk, R., Bennett, K., Bloemen, H., et al. 1996, A\&A, 315, 485

Wallace, P. M., Griffis, N. J., Bertsch, D. L., et al. 2000, ApJ, 540, 184

Address for Offprints: Marc Ribó

Service d'Astrophysique

CEA Saclay

Bât. 709, L'Orme des Merisiers

F-91191 Gif-sur-Yvette, Cedex

France 